\title{
Flavonoids and a limonoid from the fruits of Citrus unshiu and their biological activity
}

Hee Jeong Eom, ${ }^{\dagger}$ Dahae Lee,${ }^{\dagger \dagger}$ Seulah Lee, ${ }^{\dagger}$ Hyung Jun Noh,${ }^{\S}$ Jae Wook Hyun, ${ }^{\#}$ Pyoung Ho Yi, ${ }^{\#}$ Ki Sung Kang, ${ }^{\ddagger}, *$ Ki Hyun Kim ${ }^{\dagger}{ }^{*}$

${ }^{\dagger}$ School of Pharmacy, Sungkyunkwan University, Suwon 440-746, Republic of Korea

${ }^{\ddagger}$ College of Korean Medicine, Gachon University, Seongnam 461-701, Republic of Korea

${ }^{\S}$ Department of Herbal Crop Research, National Institute of Horticultural \& Herbal Science, RDA, Eumseoung 369-873, Republic of Korea

${ }^{\#}$ Citrus Research Station, National Institute of Horticultural \& Herbal Science, Rural Development Administration, Jeju 697-943, Republic of Korea

*Corresponding Author: Ki Sung Kang (Tel: +82-31-750-5402; Fax: +82-31-750-5416; E-mail: kkang@,gachon.ac.kr); Ki Hyun Kim (Tel: +8231-290-7700; Fax: +82-31-290-7730; E-mail: khkim83@skku.edu) 


\section{Contents:}

Figure S1. ${ }^{1} \mathrm{H}$ NMR spectrum of $\mathbf{1}$ in methanol- $d_{4}$

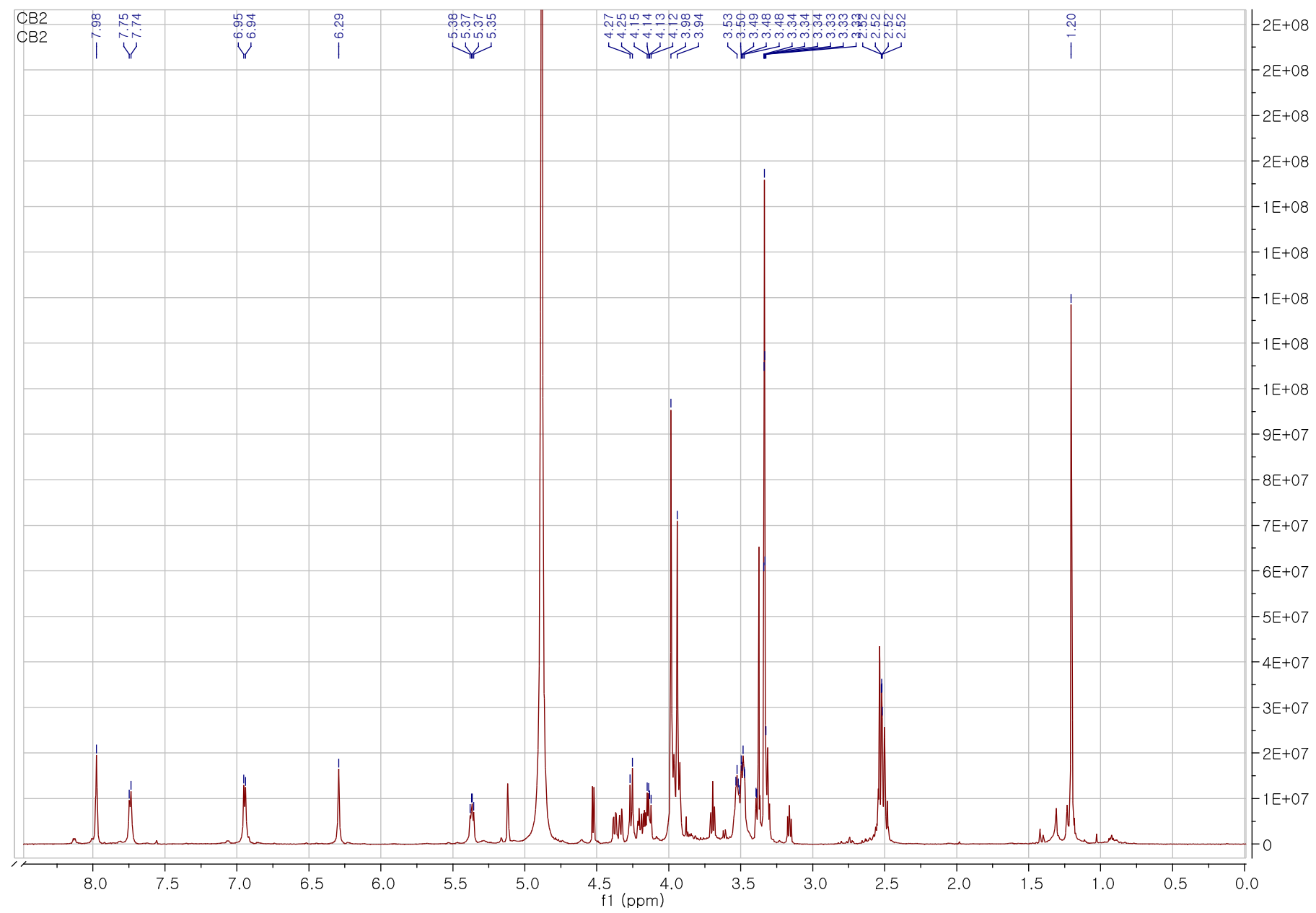


Figure S2. ${ }^{13} \mathrm{C}$ NMR spectrum of 1 in methanol- $d_{4}$

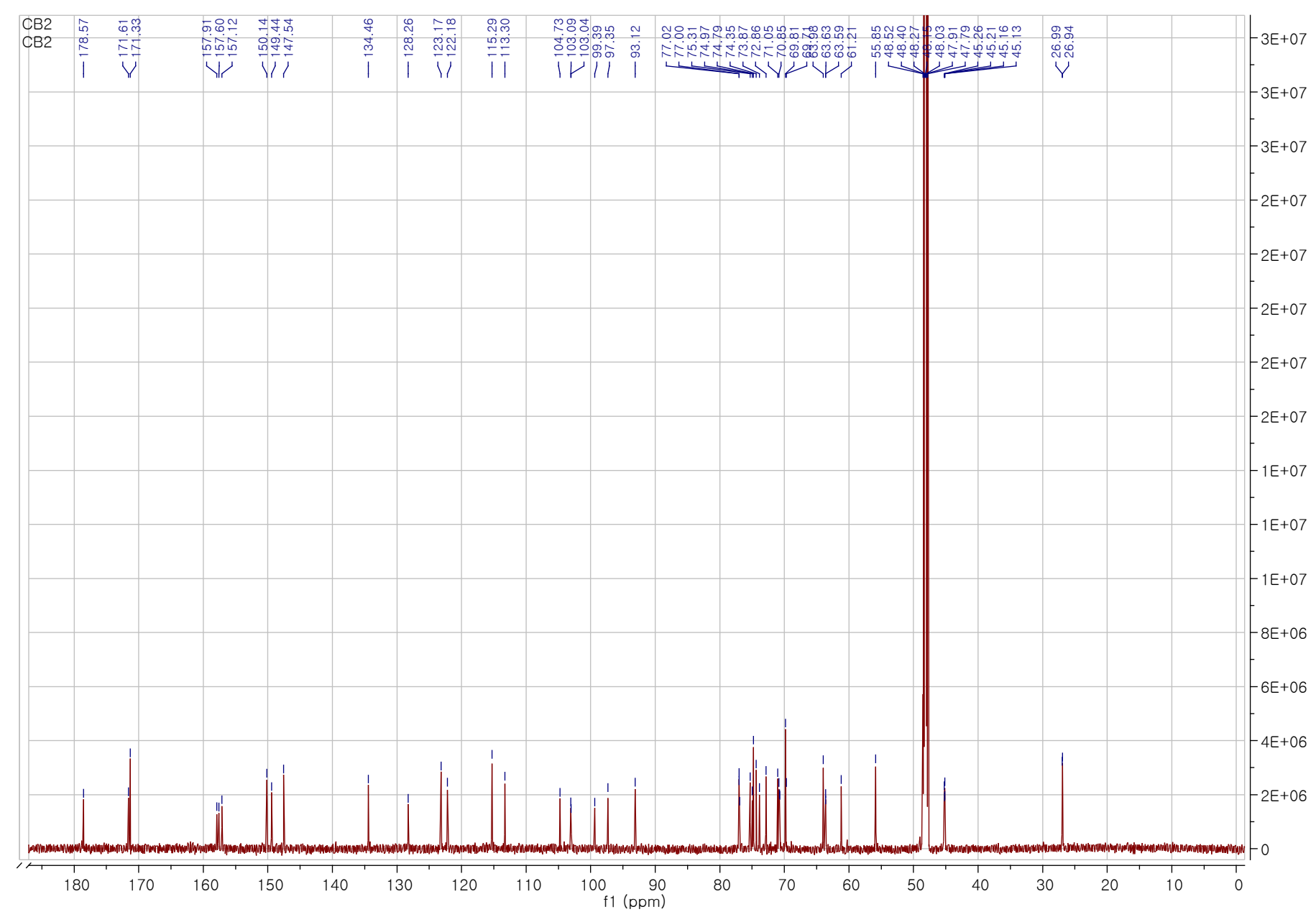


Figure S3. HSQC spectrum of $\mathbf{1}$ in methanol- $d_{4}$

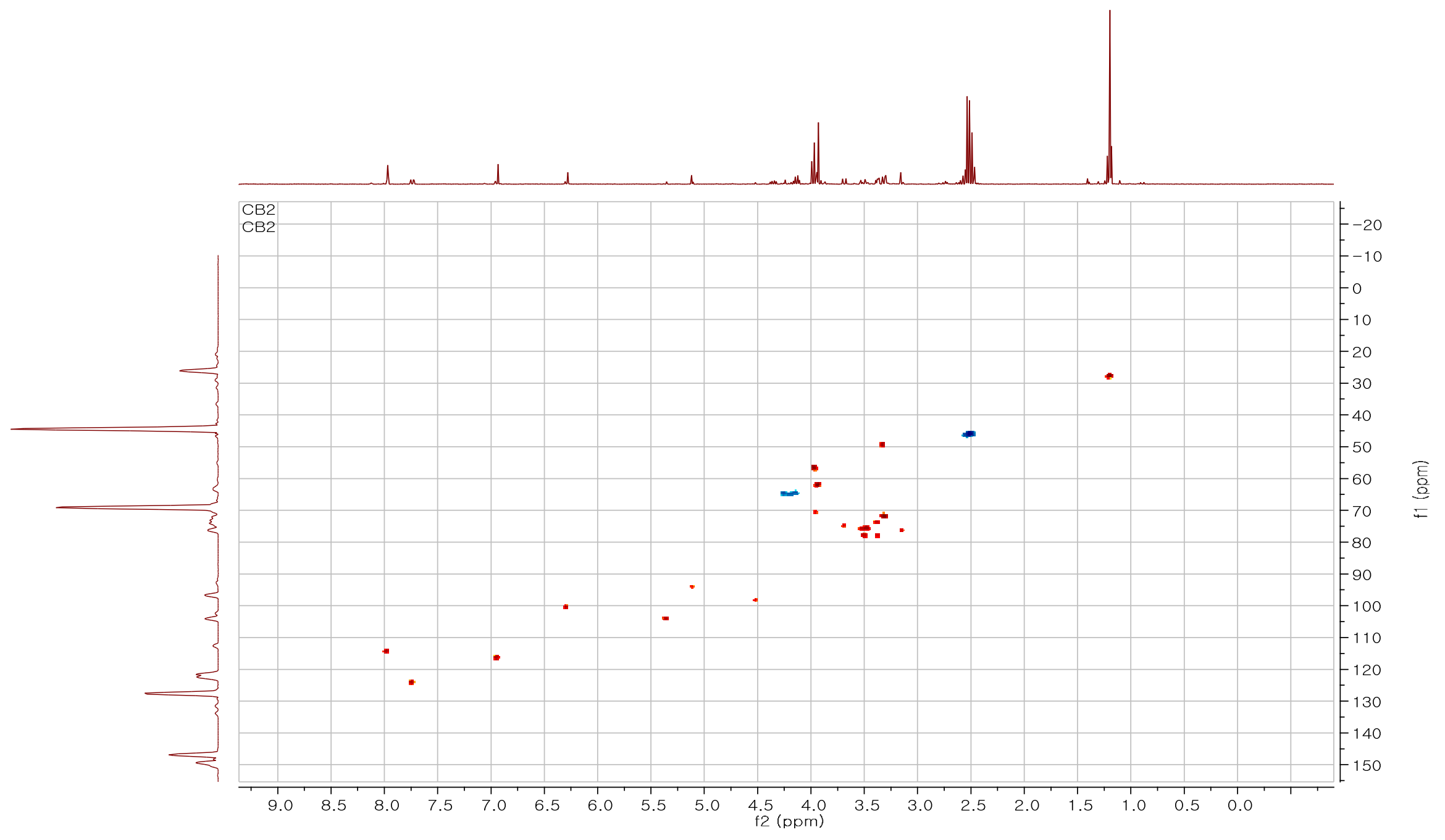


Figure S4. HMBC spectrum of $\mathbf{1}$ in methanol- $d_{4}$

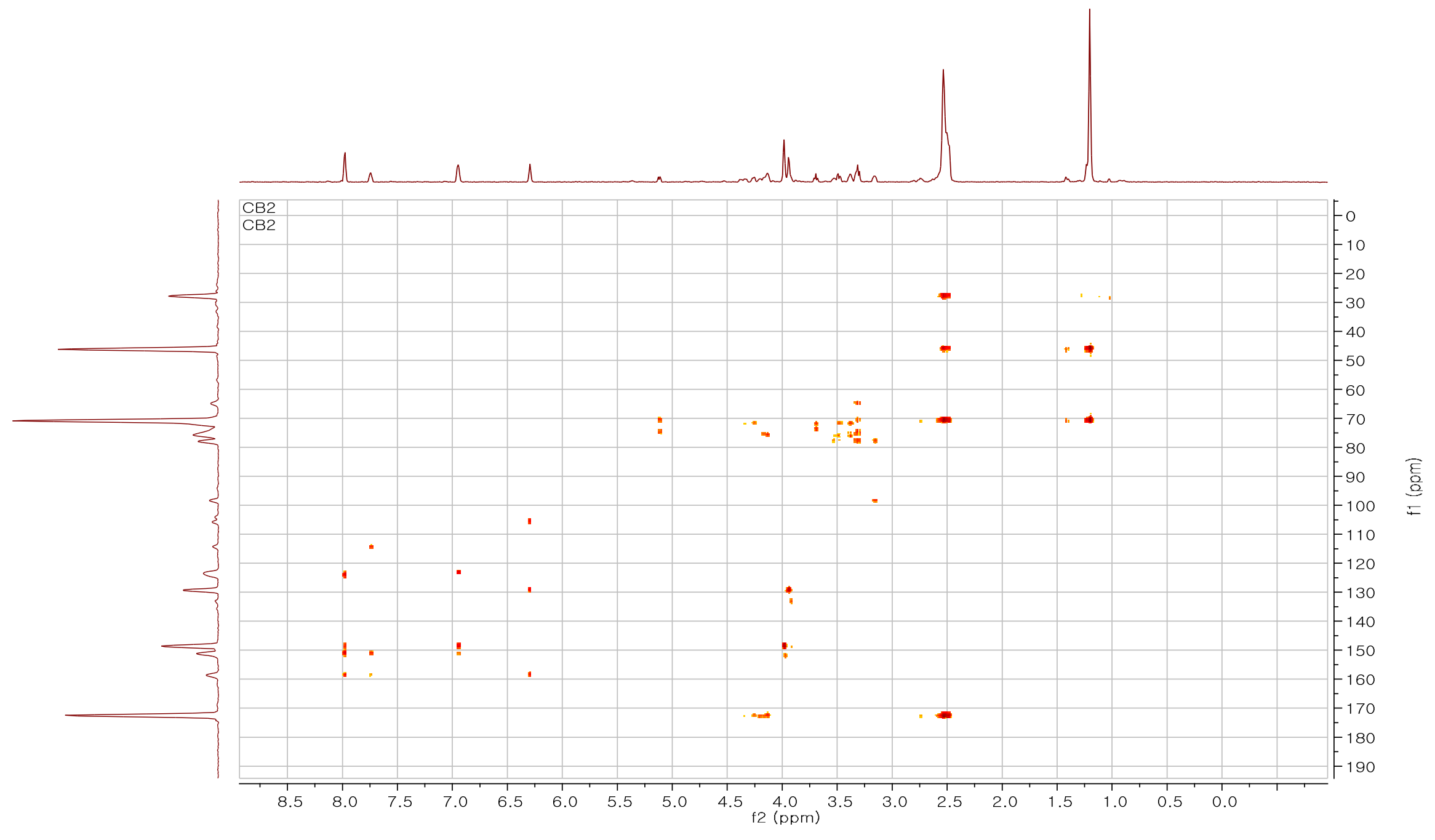


Figure S5. UV spectrum of $\mathbf{1}$ in methanol

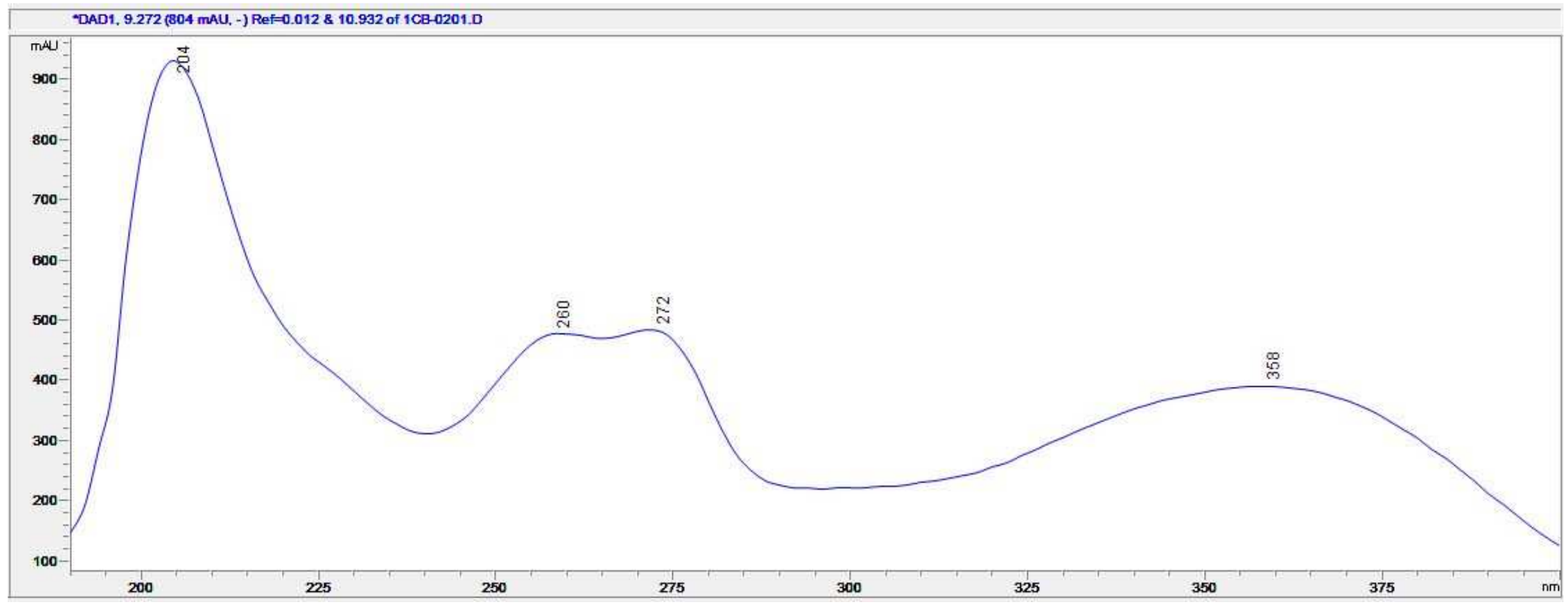


Figure S6. HR-ESI MS of 1

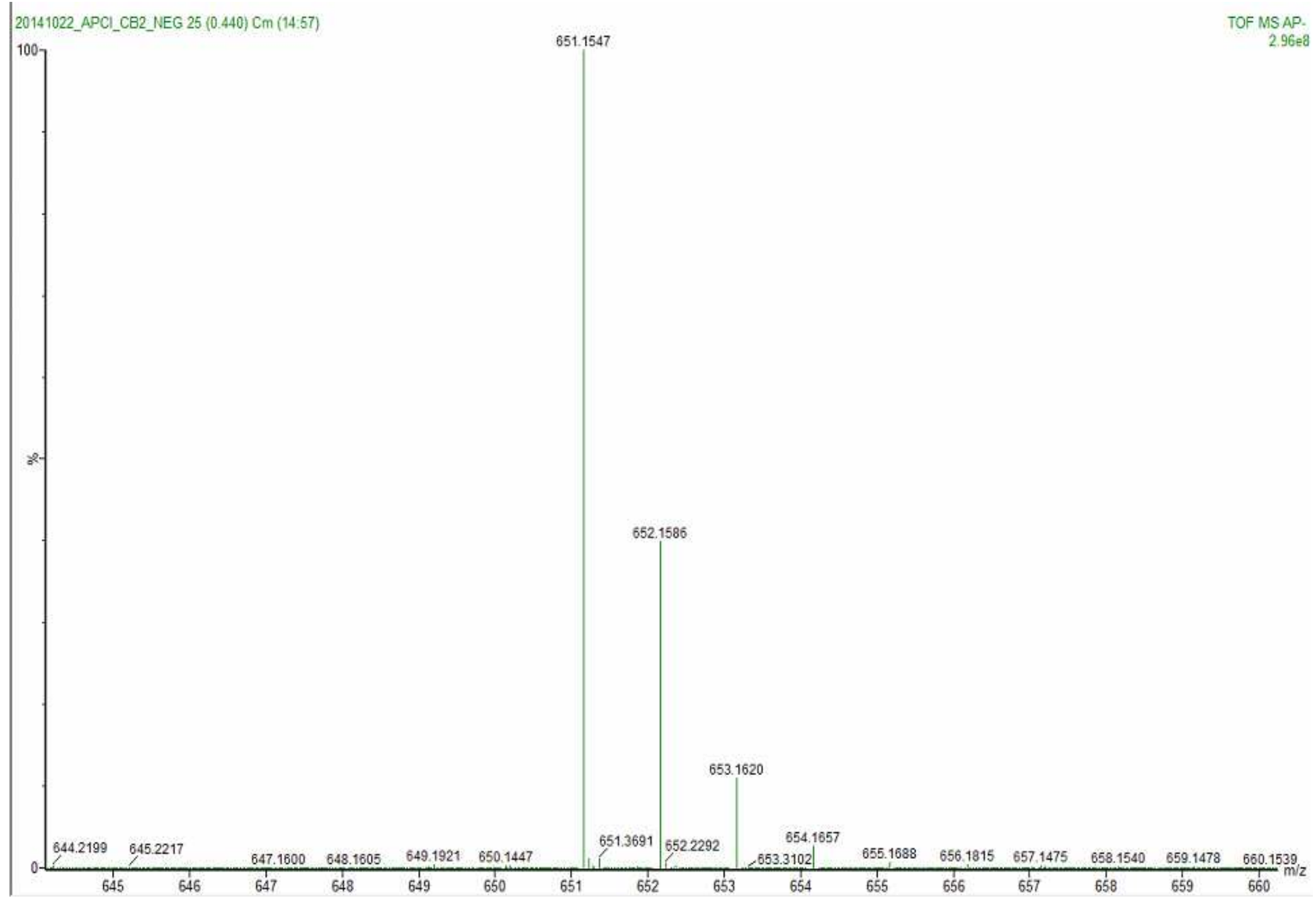




\section{Single Mass Analysis}

Tolerance $=3.0 \mathrm{PPM}$ / DBE $\min =-1.5, \mathrm{max}=50.0$

Element prediction: Off

Number of isotope peaks used for $\mathrm{i} \cdot \mathrm{FIT}=3$

Monoisotopic Mass, Odd and Even Electron lons

146 formula(e) evaluated with 1 results within limits (all results (up to 1000) for each mass)

Elements Used:

\begin{tabular}{l|c|c|c|c|l|l|l|l|l|l|l|}
\hline Mass & Cak. Mass & $\mathrm{mDa}$ & PPM & DBE & Formula & i-FIT & i-FIT Norm & Fit Conf \% & $\mathrm{C}$ & $\mathrm{H}$ & 0 \\
\hline 6511547 & 651.1561 & -1.4 & -2.2 & 14.5 & $\mathrm{C} 9 \mathrm{H} 31017$ & 2236.6 & $\mathrm{n} / \mathrm{a}$ & $\mathrm{n} / \mathrm{a}$ & 29 & 31 & 17
\end{tabular}

\begin{tabular}{lllllllllllll}
\hline 6511547 & 651.1561 & -1.4 & -2.2 & 14.5 & $\mathrm{C} 29 \mathrm{H} 31017$ & 2236.6 & $\mathrm{n} / \mathrm{a}$ & $\mathrm{n} / \mathrm{a}$ & 29 & 31 & 17
\end{tabular}

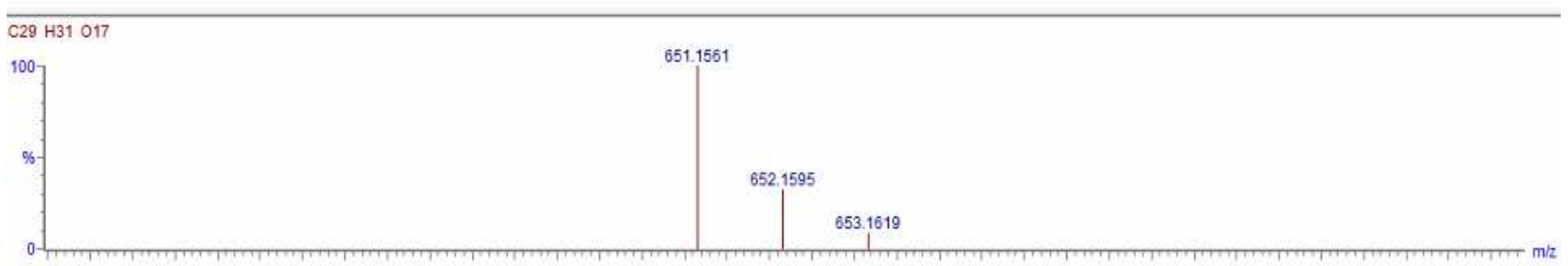

20141022_APCI_CB2_NEG $25(0.440) \mathrm{Cm}(14: 57)$ TOF MS AP.

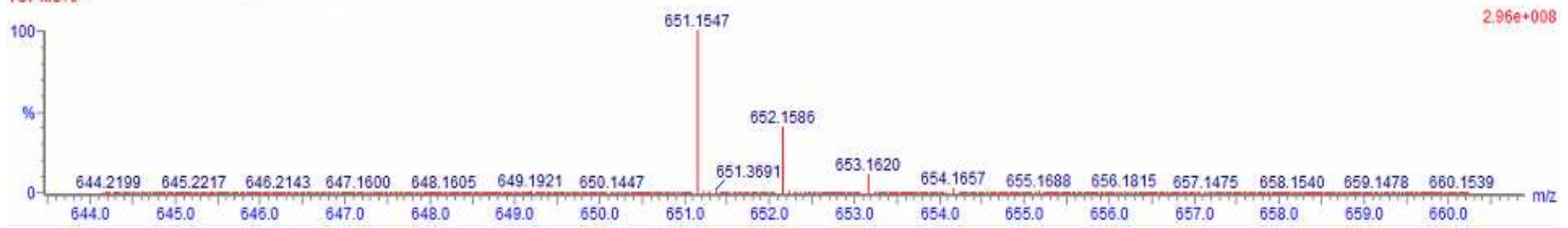

